\title{
Quantitative Characterization of Theranostic Nanoparticles by Electron Microscopy
}

\author{
M. A. Aronova ${ }^{1}$, A. A. Sousa ${ }^{2}$, R. D. Leapman ${ }^{1}$ \\ 1. National Institute of Biomedical Imaging and Bioengineering, NIH, Bethesda, MD \\ 2. Department of Biochemistry, Federal University of São Paulo, São Paulo, Brazil
}

Nanoparticles for applications in disease diagnosis and treatment have been recently the subject of intense research. These so-called theranostic nanoparticles have typically a hybrid nature; they have both organic and inorganic components. Clearly, characterization must play a central role in the design and application of such composite, hybrid theranostic nanoparticles [1].

One of the most important tools for nanoparticle characterization is transmission electron microscopy (TEM), which affords enough resolution to visualize individual nanoparticles down to the smallest sizes. Conventional TEM, however, provides no quantitative information about a given nanoparticle population other than overall particle shape and size distribution. Here we demonstrate the use of two techniques in electron microscopy for quantitative nanoparticle characterization: scanning transmission electron microscopy (STEM) [2]; and energy-filtered transmission electron microscopy / electron energy loss spectroscopy (EFTEM/EELS) [3]. For example, in the characterization of ultrasmall gold nanoparticles, STEM enables determination of the size, number of core $\mathrm{Au}$ atoms, and degree of their uniformity. STEM is also capable of determining the total molecular weight of small organic nanoparticles, such as dendrimers. EFTEM/EELS enables quantitative nanoscale mapping of specific elements in nanoparticles, such as $\mathrm{Mn}, \mathrm{Gd}$ and Fe used as contrast agents, as well as $\mathrm{C}, \mathrm{N}, \mathrm{O}$ and $\mathrm{S}$, which are often constituents of organic-based nanoparticle cores.

Fig. 1 shows an example of a manganese-block copolymer nanocomplex that contains paramagnetic Mn ions complexed with ionic-nonionic poly(ethylene oxide-b-poly(methacrylate) developed for use as a $\mathrm{T}_{1}$-weighted MRI contrast agent [4]. From the TEM image (Fig. 1a) the presence of Mn is not obvious; however, EFTEM/EELS enables mapping and quantification of manganese (Fig. 1b), and carbon (Fig. 1c) (overlaid in Fig. 1d). EELS data obtained from a single Mn containing complex is shown in Fig. 1e. After subtracting the background (red dotted line) the core edges of nitrogen, oxygen, and manganese are identifiable. Furthermore one can obtain the atomic percentage of $\mathrm{Mn}$ in the complexes, which was estimated to be $\sim 5 \%$, as shown in the histogram (Fig. 1e inset).

We have also applied these techniques to characterize other theranostic nano-delivery and imaging systems, including gold nanoparticles, dendrimers, and soft nanoparticles incorporating contrast agents such as iron oxide. [5]

References:

[1] J. B. Hall et al., Nanomedicine 2 (2007), 789.

[2] A. A. Sousa et al., J Struct Biol 159(3) (2007), 507-522.

[3] M. A. Aronova et al., J Struct Biol 160(1) (2007), 35-48.

[4] N. Pothayee et al., J. Mater. Chem. B, 40 (2) (2014), 7055-7064

[5] This research was supported by the intramural programs of NIBIB, NINDS and CC 


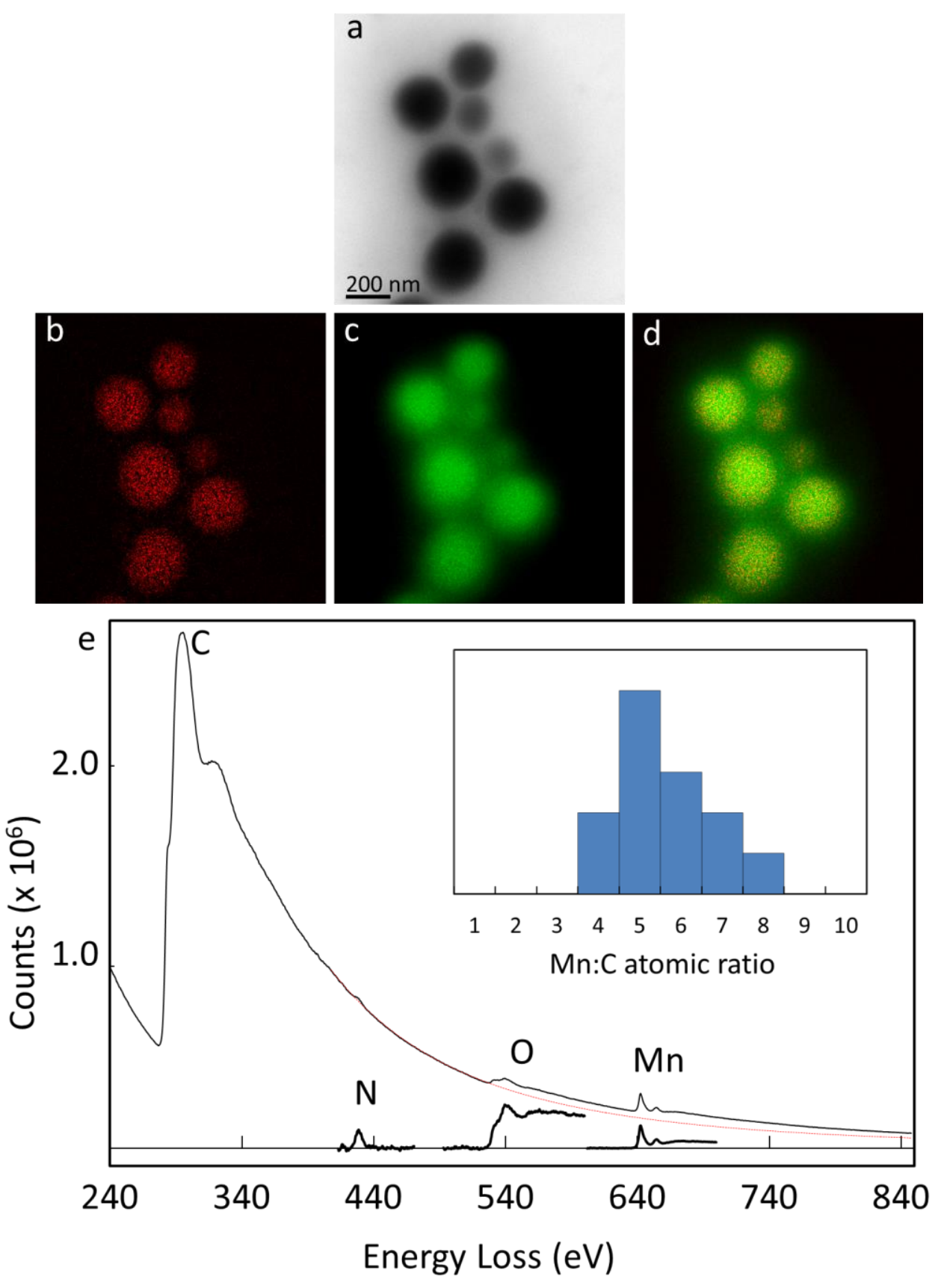

Figure 1. EFTEM and EELS analyses of manganese-block copolymer nanocomplexes. (a) TEM image of nanocomplexes, (b) manganese map (red), (c) carbon map (green), (d) overlay of manganese and carbon maps. (e) EELS spectra and background subtracted spectra obtained from the complexes showing the core edge signals of nitrogen $(\mathrm{N})$, oxygen $(\mathrm{O})$ and manganese $(\mathrm{Mn})$. From the spectra the Mn:C atomic ratios were obtained and plotted in the inset histogram. 